\title{
Patients Require Less Time to Complete Preoperative Patient-Reported Outcomes Measurement Information System (PROMIS) Than Legacy Patient- Reported Outcome Measures
}

\author{
Robert B. Browning, M.D., Thomas D. Alter, M.S., Ian M. Clapp, M.S., Nabil Mehta, M.D., \\ and Shane J. Nho, M.D., M.Sc.
}

\begin{abstract}
Purpose: To analyze time to completion of preoperative legacy patient-reported outcomes (PROs) and more recent computer adaptive Patient-Reported Outcomes Measurement Information System (PROMIS) questionnaires in patients with symptomatic femoroacetabular impingement syndrome undergoing primary hip arthroscopy. Methods: A retrospective analysis was conducted on patients undergoing hip arthroscopy by a single fellowship-trained hip arthroscopist. Inclusion criteria were patients undergoing primary arthroscopic hip surgery and completion of at least l legacy PRO or PROMIS questionnaire at the preoperative time point. Exclusion criteria were history of contralateral or ipsilateral hip surgery, non-English-speaking patients, patients who completed PROs by phone or by paper form, and patients who did not complete preoperative PROs. Legacy PROs included modified Harris Hip Score (mHHS), Hip Outcome Score (HOS), International Hip Outcome Tool (iHOT-12), and Hip Pain Visual Analog Scale (VAS-Pain). PROMIS PROs included Physical Function (PROMIS-PF), Pain Interference (PROMIS-PI), and Depression (PROMIS-D). Only preoperative PROs were included in the analysis. Completion time was calculated using the questionnaire start and stop time reported by the survey collecting software. The median and interquartile range of each PRO were reported for analysis of central tendency and statistical dispersion, respectively. Results: A total of 1,901 patients and 269 patients were included in the legacy and PROMIS groups, respectively. The median time required for completion of each PRO in (minutes: seconds) format was as follows: mHHS (1:29), HOS (3:58), iHOT (2:11), VAS-Pain (0:32), PROMIS-PF (0:46), PROMIS-PI (0:37), and PROMIS-D $(0: 43)$. The interquartile range of the middle $50 \%$ of respondents was as follows; mHHS (0:58), HOS (2:46), iHOT (1:22), VAS-Pain (0:28), PROMIS-PI (0:19), PROMIS-D (0:29), and PROMIS-PF (0:20). Conclusions: This study supports that preoperative PROMIS forms require less time to complete than preoperative legacy PROs and are not significantly influenced by age, race, or workers compensation status. Level of Evidence: Level IV, case series.
\end{abstract}

Section of Young Adult Hip Surgery, Division of Sports Medicine, Department of Orthopedic Surgery, Hip Preservation Center, Rush University Medical Center, Chicago, Illinois, U.S.A.

The authors report the following potential conflicts of interest or sources of funding: S.J.N. reports nonfinancial support from AlloSource, other from the American Orthopaedic Association and American Orthopaedic Society for Sports Medicine, nonfinancial support from Arthrex, other from Arthroscopy Association of North America, nonfinancial support from Athletico, DJ Orthropaedics, ConMed Linvatec, and MioMed, personal fees from Ossur, nonfinancial support from Smith $\theta$ Nephew, and personal fees from Springer and Stryker, outside the submitted work. Full ICMJE author disclosure forms are available for this article online, as supplementary material.

Received January 11, 2021; accepted June 25, 2021.

Address correspondence to Thomas D. Alter, M.Sc., Department of Orthopedic Surgery, Rush University Medical Center, Chicago, IL 60612. E-mail: thomas.alter137@gmail.com

(C) 2021 THE AUTHORS. Published by Elsevier Inc. on behalf of the Arthroscopy Association of North America. This is an open access article under the CC BY-NC-ND license (http://creativecommons.org/licenses/by-nc-nd/4.0/).

2666-061X/2137

https://doi.org/10.1016/j.asmr.2021.06.011
$\mathbf{T}$ he drive to improve efficiency in patient-reported outcome (PRO) collection and delivery stems from a will to improve compliance and accuracy of information. Legacy PROs including Hip Outcome Score - Activities of Daily Living (HOS-ADL), ${ }^{1}$ Hip Outcome Score - Sports Subscale (HOS-SS), ${ }^{2}$ modified Harris Hip Score (mHHS), ${ }^{3}$ International Hip Outcome Tool (iHOT-12), ${ }^{4}$ and visual analog scale for pain (VAS Pain) traditionally have been used to evaluate preoperative hip function and postoperative clinical outcomes in patients undergoing hip arthroscopy. In 2004, The National Institutes of Health developed the PatientReported Outcomes Measurement Information System (PROMIS) with the goal of providing a singular, standardized outcomes measure that can be applied to a wide variety of conditions. To alleviate complications associated with multiple, noncomparable PROs, 
PROMIS has added integrated response theory (IRT) with computerized adaptive testing (CAT), which has led to the development of a single, validated, generalizable system for assessing PROs. ${ }^{5,6}$ With the addition of these measures, PROMIS has reduced redundant questions while addressing multiple modalities, therefore reducing the number of responses required to provide an outcome score assessment in comparison with legacy PROs, while providing accurate health state estimations across specialties. ${ }^{7,8}$ PROMIS also has been shown to be an effective and efficient tool for reporting patient outcomes in various orthopaedic surgery procedures $^{9-11}$ and has strongly correlated with common legacy PROs. ${ }^{12,13}$

It is well established within the literature that hip arthroscopy is an effective treatment for femoroacetabular impingement syndrome (FAIS) and leads to improvement in PROs over time. ${ }^{14-21}$ A series of legacy PROs often are used to evaluate patient functional status and pain but are not without limitations. PROs often are administered in the physician's office before appointments, which can delay care and lead to inefficiencies. Excessive and redundant questions can cause questionnaire burnout and lead to inaccurate or faulty data, limiting its clinical and research value. In addition, burdensome PROs may limit willingness to complete forms following surgery. Recently, PROMIS CAT forms have been used for measuring functional status in the hip arthroscopy population and have shown to have excellent correlation with legacy hip PROs, establishing itself as a useful adjunct and possible alternative to traditional outcome measures in patients with FAIS. ${ }^{22-24}$ PROMIS PROs have demonstrated improved average time to completion (TTC) in patients undergoing cervical spine ${ }^{25}$ and upper extremity ${ }^{26}$ surgery; however, much remains unknown about its effect on the efficiency of questionnaire delivery in patients undergoing hip arthroscopy.

The purpose of this study is to analyze time to completion of preoperative legacy PROs and more recent computer adaptive PROMIS questionnaires in patients with symptomatic FAIS undergoing primary hip arthroscopy. We hypothesized that PROMIS PROs would require less TTC when compared with legacy PROs, and that PROMIS PROs would display less variability in TTC across patients undergoing hip arthroscopy.

\section{Methods}

\section{Patient Selection}

After institutional review board approval, a prospectively collected database of patients undergoing hip arthroscopy for FAIS by a single surgeon between November 2013 and July 2019 was retrospectively analyzed. Inclusion criteria were patients undergoing primary arthroscopic hip surgery and completion of at least 1 legacy PRO or PROMIS questionnaire at the preoperative time point. Exclusion criteria were history of contralateral or ipsilateral hip surgery, non-Englishspeaking patients, patients who completed PROs by phone or by paper form, and patients who did not complete at least 1 preoperative legacy PRO or at least 1 PROMIS questionnaire. Postoperative PROs were not included in this study. Legacy PROs included mHHS, HOS, iHOT-12, and VAS-Pain, whereas PROMIS questionnaires included Physical Function (PROMIS-PF), version 2.0, Pain Interference (PROMIS-PI), version 1.1, and Depression (PROMIS-D), version 1.0.

\section{Data Collection}

Legacy PROs were collected between November 2013 and December 2017, and PROMIS scores were collected from their introduction at our institution in January 2018 to July 2019. All surveys collected were administered before the surgical date at the initial visit using a table device (iPad tablet; Apple, Cupertino, CA). All PROs were electronically collected and stored on the OBERD data collection platform (Universal Research Solutions, Columbia, MO). The outcomes of interest were the TTC each individual PRO. The raw data export from OBERD contains a start and stop time stamp for PROs completed by each patient. The completion time was determined by calculating the difference between the questionnaire start and stop time reported by the survey collecting software. ${ }^{27}$ Patient demographics also were collected, including age, sex, race, ethnicity, and workers compensation status.

\section{Statistical Analysis}

The Shapiro-Wilk test was used to determine normality and box plots were used to identify outliers. All PRO datasets were non-normal, right skewed, and contained outliers. As such, the interquartile range (IQR) method was used to identify outliers, with limits placed at 1.5 IQR from the first and third quartile. Cases outside of these limits were removed from subsequent analysis. ${ }^{28}$ The median and IQR of each PRO were reported for analysis of central tendency and statistical dispersion, respectively. In addition, the number of questions required to complete each PRO was determined.

All data were inspected before analysis to determine whether parametric statistical analysis assumptions were met. The Shapiro-Wilk test was used to determine normality. The Levene test was performed to assess for equality of variances. For continuous variables with 2 groups, independent sample $t$-test or Mann-Whitney $U$ test was used. For continuous variables with more than 2 groups, analysis of variance or Kruskal-Wallis test were used. Categorical variables were performed to determine significant differences in 
Table 1. Percent of Cases Within Outlier Limits After IQR Outlier Removal

\begin{tabular}{lc}
\hline Legacy & \\
mHHS & $85.7 \%$ \\
HOS & $88.3 \%$ \\
iHOT-12 & $86.8 \%$ \\
Pain & $80.4 \%$ \\
PROMIS & \\
PI & $88.8 \%$ \\
D & $91.0 \%$ \\
PF & $90.6 \%$ \\
\hline
\end{tabular}

D, PROMIS Depression; HOS, Hip Outcome Score; iHOT-12, international Hip Outcome Tool; IQR, interquartile range; mHHS, modified Harris Hip Score; Pain, Hip Pain visual analog scale; PF, PROMIS Physical Function; PI, PROMIS Pain Interference; PROMIS, PatientReported Outcomes Measurement Information System.

categorical variables. All statistical testing was performed in SPSS, version 26 (IBM Corp., Armonk, NY).

\section{Results}

\section{Time to Completion}

A total of 1,901 patients and 269 patients completed at least 1 legacy PRO and at least 1 PROMIS subscore at the preoperative time point, respectively. The percentage of patients completing each preoperative PRO is as follows: mHHS $(83.2 \%)$, HOS $(79.0 \%)$, iHOT- 12 $(84.6 \%)$, VAS for pain $(76.2 \%)$, PROMIS-PI $(89.2 \%)$, PROMIS-D (91.4\%), and PROMIS-PF (91.4\%). The IQR method was used before subsequent analysis, identifying a greater percentage of outliers in the legacy PROs in comparison with PROMIS PROs (Table 1). The average number of questions required to complete PROMIS PROs was less than all legacy PROs, with the exception being VAS-Pain (Table 2). The median TTC (minutes: seconds) of each PRO was as follows: mHHS (1:29), HOS (3:58), iHOT (2:11), VAS-Pain (0:32), PROMIS-PI (0:37), PROMIS-D (0:43), and PROMIS-PF $(0: 46)$ (Fig 1). The IQR of the middle $50 \%$ of respondents was as follows: mHHS (0:58), HOS (2:46), iHOT (1:22), VAS-Pain (0:28), PROMIS-PI (0:19), PROMIS-D (0:29), and PROMIS-PF (0:20).

\section{Demographics: Legacy Versus PROMIS Groups}

A $\chi^{2}$ analysis of the demographics of patients completing legacy and PROMIS PROs indicated similar group composition in all patient demographics except sex. Patients completing PROMIS PROs was $77.2 \%$ female, whereas legacy PROs contained $71.2 \%$ female subjects (Table 3 ).

\section{Demographic: TTC}

Analysis of completion time stratified by age indicated significant differences in TTC between age groups for all legacy PROs $(P<.05$; Table 4$)$. Planned post hoc comparison was performed on each individual legacy
PRO comparing TTC between 2 individual age groups at a time. All legacy PROs at each age group had at least one significant difference in TTC on post hoc testing. The Shapiro-Wilk test for PRO completion times indicated non-normal distribution. As such, Spearman rank correlation analysis was performed between age and TTC for each PRO. Significant positive correlations were found between age and time to completion in all legacy PROs (Table 4). No significant correlations were found between age and time to completion in PROMIS PROs.

An analysis stratified by race indicated significant differences in TTC between groups in HOS and iHOT12 , and VAS for pain $(P<.001$ for all; Table 4$)$. Planned post hoc comparison of HOS, iHOT-12, and VAS for pain indicated that African American patients had a greater TTC in comparison with White and Asian patients $(P<.01)$. Mann-Whitney $U$ analysis indicated that patients of Hispanic or Latino ethnicity required a greater amount of time to complete mHHS, HOS, PROMIS-PF $(P<.05)$ than non-Hispanic patients (Table 4). Mann-Whitney $U$ analysis of sex indicated that female subjects had a greater TTC for HOS $(P=.012)$ in comparison with their male counterparts (Table 4). Patients receiving workers compensation had greater TTC for mHHS, HOS, iHOT-12, and VAS for pain $(P<.01$; Table 4$)$.

\section{Discussion}

In this study, we found that PROMIS PROs required less TTC, demonstrated reduced variability in TTC, necessitated fewer questions to complete, and displayed less variability across patient demographics than legacy PROs. This study demonstrated that the majority of patients undergoing hip arthroscopy are able to complete PROMIS-specific questionnaires in less than 1 minute, which is consistent with other patient populations..$^{29-32}$ In comparison, the median TTC of individual legacy PROs ranged from 30 seconds in VASPain to nearly 4 minutes for the HOS questionnaire.

Table 2. Number of Questions Required to Complete Legacy and PROMIS PROs

\begin{tabular}{lr}
\hline Legacy & \\
mHHS & 8 \\
HOS & 31 \\
iHOT-12 & 12 \\
Pain & 1 \\
PROMIS & $4.1 \pm 0.7$ \\
PI & $5.6 \pm 2.8$ \\
D & $4.0 \pm 0.2$ \\
PF & \\
\hline D, PROMIS Depression; HOS, Hip Outcome Score; iHOT-12, inter- \\
national Hip Outcome Tool; mHHS, modified Harris Hip Score; Pain, \\
Hip Pain visual analog scale; PF, PROMIS Physical Function; PI, \\
PROMIS Pain Interference; PROMIS, Patient-Reported Outcomes \\
Measurement Information System.
\end{tabular}




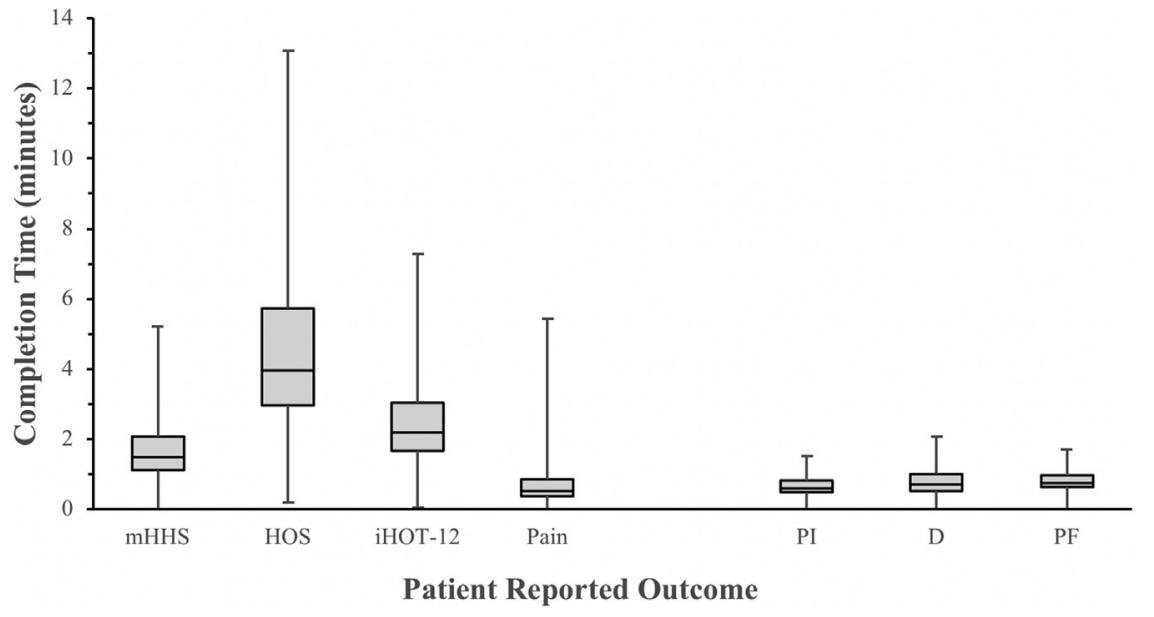

Fig 1. Completion time to completion of legacy and novel patient-reported outcomes (in minutes). The box plots depict the minimum, first quartile, median, third quartile, and maximum completion time. (D, PROMIS Depression; HOS, Hip Outcome Score; iHOT, international Hip Outcome Tool; mHHS, modified Harris Hip Score; Pain, Hip Pain visual analog scale; PF, PROMIS Physical Function; PI, PROMIS Pain Interference; PROMIS, Patient-Reported Outcomes Measurement Information System.)
In addition, PROMIS PROs required fewer total questions to complete than legacy PROs.

It is well-established within the literature that waiting time is negatively correlated with patient satisfaction in the outpatient orthopaedic setting ${ }^{3-36}$ and that a reduction in wait time leads to improved patient satisfaction. ${ }^{37}$ PROs have provided clinicians with useful, quantitative data to determine the efficacy of various treatments within orthopaedic surgery and beyond. ${ }^{38-40}$ To ensure accuracy of information, patient compliance and reduction of questionnaire burnout, PRO measures must be short, concise, and simple to answer. The implementation of a shorter, quicker, and more predictable PRO system could potentially lead to more accurate patient appointment slots, shorter waiting times, improved patient satisfaction, and ultimately improved accuracy of patient outcome information in busy orthopedic practices.

Previous studies have evaluated the TTC of PROMIS CAT in multiple contexts. ${ }^{25-27}$ Kadri et al. ${ }^{27}$ evaluated older cohort (age: 47 years; race: 65\% White, 19.4\% African American) undergoing cervical spine surgery and reported a mean TTC of PI (1.05 minutes), PF (0.74 minutes), and D (0.57 minutes). Interestingly, examination of race demonstrated the longest and shortest TTC in Asian and White patients, respectively. In addition, Hispanic patients demonstrated the shortest TTC in comparison with non-Hispanic patients. ${ }^{27}$ Iyer et al. ${ }^{25}$ evaluated an older cohort (age: 57 years; race: $92 \%$ White) undergoing cervical spine surgery and reported a median TTC of PI (29.5 seconds) and PF (37.0 seconds). In addition, Tyser et al. $^{26}$ evaluated patients undergoing upper-extremity surgery (age: 42 years; race: $92 \%$ White) and reported an average PF of 57 seconds. Similarly, in a study consisting of 2,952 operative and nonoperative patients (age: 51.0 years), Gulledge et al. ${ }^{10}$ reported a mean TTC and number of questions necessary to complete PROMIS-UE,
PROMIS-PF, and PROMIS-PI ranging from 45.3 to 54.4 seconds and 4.1 to 4.9 questions, whereas PROMIS-D ranged from 20.9 to 38.6 seconds for all groups and required 6.2 to 6.7 questions to complete. These results are similar to the findings of the present study; however, it is important to note that patient age, race, and the reporting of central tendency may influence the reported average TTC in completion of PROMIS forms.

The findings in the present study support that PROMIS PROs have less variability in TTC than legacy PROs. The IQR, defined as the difference in TTC for the

Table 3. Demographics of Patients' Complete Legacy and PROMIS PROs

\begin{tabular}{|c|c|c|c|}
\hline & $\begin{array}{c}\text { Legacy } \\
(\mathrm{n}=1901)\end{array}$ & $\begin{array}{l}\text { PROMIS } \\
(\mathrm{n}=269)\end{array}$ & $P$ Value \\
\hline \multicolumn{4}{|l|}{ Age, y } \\
\hline$<20$ & $23.7 \%$ & $26.0 \%$ & \multirow[t]{5}{*}{.195} \\
\hline $20-30$ & $22.4 \%$ & $25.7 \%$ & \\
\hline $30-40$ & $23.8 \%$ & $25.3 \%$ & \\
\hline $40-50$ & $16.6 \%$ & $13.0 \%$ & \\
\hline$>50$ & $13.7 \%$ & $10.0 \%$ & \\
\hline \multicolumn{4}{|l|}{ Sex } \\
\hline Male & $28.8 \%$ & $22.8 \%$ & \multirow[t]{2}{*}{$.040^{*}$} \\
\hline Female & $71.2 \%$ & $77.2 \%$ & \\
\hline \multicolumn{4}{|l|}{ Race } \\
\hline White & $93.4 \%$ & $92.7 \%$ & \multirow[t]{4}{*}{.332} \\
\hline Black & $3.5 \%$ & $2.7 \%$ & \\
\hline Asian & $2.0 \%$ & $2.3 \%$ & \\
\hline Other & $1.1 \%$ & $2.3 \%$ & \\
\hline \multicolumn{4}{|l|}{ Ethnicity } \\
\hline Not Hispanic or Latino & $92.5 \%$ & $93.0 \%$ & \multirow[t]{2}{*}{0.765} \\
\hline Hispanic or Latino & $7.5 \%$ & $7.0 \%$ & \\
\hline Workers compensation & $2.8 \%$ & $2.8 \%$ & 0.994 \\
\hline \multicolumn{4}{|c|}{$\begin{array}{l}\text { D, PROMIS Depression; HOS, Hip Outcome Score; iHOT-12, inter- } \\
\text { national Hip Outcome Tool; IQR, interquartile range; mHHS, modified } \\
\text { Harris Hip Score; Pain, hip pain visual analog scale; PF, PROMIS } \\
\text { Physical Function; PI, PROMIS Pain Interference; PROMIS, Patient- } \\
\text { Reported Outcomes Measurement Information System. } \\
\text { *Indicates statistically significant }(P<.05) \text { between groups. }\end{array}$} \\
\hline
\end{tabular}


Table 4. Preoperative PRO Completion Time (Minutes: Seconds) by Demographic

\begin{tabular}{|c|c|c|c|c|c|c|c|}
\hline & \multicolumn{4}{|c|}{ Legacy $(n=1901)$} & \multicolumn{3}{|c|}{ PROMIS $(\mathrm{n}=269)$} \\
\hline & mHHS & HOS & iHOT-12 & Pain & PI & D & $\mathrm{PF}$ \\
\hline \multicolumn{8}{|l|}{ Age, y } \\
\hline$<20$ & $01: 44$ & 04:44 & $02: 27$ & $00: 55$ & $00: 44$ & $00: 51$ & $00: 52$ \\
\hline $20-30$ & $01: 38$ & $04: 23$ & $02: 29$ & $00: 46$ & $00: 37$ & $00: 44$ & $00: 44$ \\
\hline $30-40$ & $01: 41$ & $04: 35$ & $02: 28$ & $00: 50$ & $00: 40$ & $00: 50$ & $00: 52$ \\
\hline $40-50$ & $01: 48$ & $04: 55$ & $02: 34$ & 00:49 & $00: 38$ & $00: 48$ & $00: 50$ \\
\hline$>50$ & $01: 59$ & $04: 58$ & $02: 53$ & 01:02 & 00:39 & $00: 53$ & $00: 50$ \\
\hline Male & $01: 44$ & $04: 26^{\dagger}$ & $02: 32$ & $00: 51$ & $00: 37$ & $00: 48$ & $00: 48$ \\
\hline Female & $01: 44$ & $04: 45^{\dagger}$ & $02: 32$ & $00: 52$ & $00: 41$ & $00: 49$ & $00: 49$ \\
\hline \multicolumn{8}{|l|}{ Race } \\
\hline White & $01: 43$ & $04: 34$ & $02: 30$ & $00: 50$ & $00: 40$ & $00: 48$ & $00: 49$ \\
\hline Black & 01:59 & $06: 29$ & $03: 18$ & 01:09 & $00: 35$ & $00: 54$ & $00: 41$ \\
\hline Asian & $01: 40$ & $04: 42$ & $02: 29$ & $00: 44$ & $00: 37$ & $00: 45$ & $00: 58$ \\
\hline \multicolumn{8}{|l|}{ Workers compensation } \\
\hline Yes & $02: 30^{*}$ & $06: 10^{*}$ & $03: 18^{*}$ & $01: 15^{*}$ & $00: 48$ & $00: 47$ & $00: 55$ \\
\hline No & $01: 42^{*}$ & $04: 40^{*}$ & $02: 28^{*}$ & $00: 49^{*}$ & $00: 38$ & $00: 47$ & $00: 48$ \\
\hline
\end{tabular}

NOTE. Values are presented as means unless otherwise noted.

D, PROMIS Depression; HOS, Hip Outcome Score; iHOT-12, international Hip Outcome Tool; IQR, interquartile range; mHHS, modified Harris Hip Score; Pain, hip pain visual analog scale; PF, PROMIS Physical Function; PI, PROMIS Pain Interference; PRO, patient-reported outcome; PROMIS, Patient-Reported Outcomes Measurement Information System.

*Indicates time to completion that are involved in a statistically significant outcomes $(P<.05)$ between groups. Statistically significant difference in completion time compared by patient age and race are not depicted in the table.

25th and 75th percentile, for completion of legacy PROs ranged from 28 seconds for VAS-Pain, which is a single question survey, to just less than 3 minutes for HOS. The IQR for each of the PROMIS forms was less than 30 seconds. These results are supported by previous studies evaluating the variability in TTC for PROMIS forms. ${ }^{29,41}$ In addition, the IQR of patients completing PROMIS forms was less than 30 seconds, and there was no difference in TTC based on age, sex, race, or workers compensation status. Significant differences were seen in TTC for legacy PROs based on age, sex, race, ethnicity and workers compensation status. Patients older than 50 years of age completed each of the legacy PROs slower than their younger counterparts, which is consistent with previous studies using electronic software for data collection. ${ }^{27,42}$ African Americans completed legacy PROs slower than that of White and Asian ethnicity, female subjects took longer to complete HOS than male subjects, and patients with workers compensation injuries took significantly longer to complete mHHS, HOS, iHOT-12, and VAS for pain forms. These results differ from that of a recent study by Kadri et al., ${ }^{27}$ which found that PROMIS forms were completed fastest by young patients and those of Hispanic ethnicity.

\section{Limitations}

The present study has limitations that are worth noting. Time required to complete each of the PROMIS and legacy PROs is intrinsically right skewed and further skewed, in part, due to a number of patients failing to complete the questionnaires in one sitting, without interruption. For this reason, reporting the median TTC and the IQR provides a more accurate representation of average time to complete the PROMIS and legacy PROs in this population. ${ }^{43}$ In addition, our institution uses multiple PRO collection modalities, including OBERD (Columbia, MO), phone calls, and written forms. Patient who completed the questionnaires over the phone or on paper may be less comfortable with technology, leading to our study potentially misrepresenting the true population. Moreover, we did not collect the order in which each PRO was completed. If questionnaire burnout influences response rate, patients may complete later questionnaires faster (with less care) or possibly slower due to lack of motivation or attention. In addition, while most questionnaires were completed at the time of clinic visit, some patients completed surveys at another time outside the clinic but before surgery. Finally, our current study was performed at a single 
tertiary care institution and may not be representative of the entire hip arthroscopy population. Future studies are warranted to determine the utility of PROMIS forms in assessing clinical outcomes in patients undergoing hip arthroscopy. Specifically, the relationship between preoperative and postoperative PRO completion time and achievement of clinically significant outcome may be of value.

\section{Conclusions}

This study supports that preoperative PROMIS forms require less time to complete than preoperative legacy PROs and are not significantly influenced by age, race, or workers compensation status.

\section{References}

1. Martin RL, Kelly BT, Philippon MJ. Evidence of validity for the hip outcome score. Arthroscopy 2006;22: 1304-1311.

2. Martin RL, Philippon MJ. Evidence of reliability and responsiveness for the hip outcome score. Arthroscopy 2008;24:676-682.

3. Byrd JW. Hip arthroscopy: Patient assessment and indications. Instr Course Lect 2003;52:71 1-719.

4. Griffin DR, Parsons N, Mohtadi NG, Safran MR. Multicenter Arthroscopy of the Hip Outcomes Research N. A short version of the International Hip Outcome Tool (iHOT-12) for use in routine clinical practice. Arthroscopy 2012;28:611-616. quiz 616-618.

5. Cella D, Gershon R, Lai JS, Choi S. The future of outcomes measurement: Item banking, tailored short-forms, and computerized adaptive assessment. Qual Life Res 2007;16: 133-141 (Suppl 1).

6. Chang $\mathrm{CH}$, Reeve $\mathrm{BB}$. Item response theory and its applications to patient-reported outcomes measurement. Eval Health Prof 2005;28:264-282.

7. Fries JF, Witter J, Rose M, Cella D, Khanna D, MorganDeWitt E. Item response theory, computerized adaptive testing, and PROMIS: Assessment of physical function. J Rheumatol 2014;41:153-158.

8. Wylie JD, Beckmann JT, Granger E, Tashjian RZ. Functional outcomes assessment in shoulder surgery. World $J$ Orthop 2014;5:623-633.

9. Bernstein DN, Houck JR, Hammert WC. A Comparison of PROMIS UE versus PF: Correlation to PROMIS PI and Depression, ceiling and floor effects, and time to completion. J Hand Surg Am 2019;44:901.e901-901.e907.

10. Gulledge CM, Smith DG, Ziedas A, Muh SJ, Moutzouros V, Makhni EC. Floor and ceiling effects, time to completion, and question burden of PROMIS CAT domains among shoulder and knee patients undergoing nonoperative and operative treatment. JB JS Open Access 2019;4:e0015.1-e0015.7.

11. Anthony CA, Glass NA, Hancock K, Bollier M, Wolf BR, Hettrich CM. Performance of PROMIS instruments in patients with shoulder instability. Am J Sports Med 2017;45:449-453.

12. Fidai MS, Saltzman BM, Meta F, et al. Patient-reported outcomes measurement information system and legacy patient-reported outcome measures in the field of orthopaedics: A systematic review. Arthroscopy 2018;34: 605-614.

13. Li DJ, Clohisy JC, Schwabe MT, Yanik EL, PascualGarrido C. PROMIS versus legacy patient-reported outcome measures in patients undergoing surgical treatment for symptomatic acetabular dysplasia. Am J Sports Med 2020;48:385-394.

14. Beck EC, Nwachukwu BU, Chahla J, et al. Patients with borderline hip dysplasia achieve clinically significant outcome after arthroscopic femoroacetabular impingement surgery: A case-control study with minimum 2-year follow-up. Am J Sports Med 2019;47:2636-2645.

15. Bozic KJ, Chan V, Valone FH 3rd, Feeley BT, Vail TP. Trends in hip arthroscopy utilization in the United States. J Arthroplasty 2013;28:140-143.

16. Cancienne JM, Beck EC, Kunze KN, Chahla J, Suppauksorn S, Nho SJ. Functional and clinical outcomes of patients undergoing revision hip arthroscopy with borderline hip dysplasia at 2-year follow-up. Arthroscopy 2019;35:3240-3247.

17. Levy DM, Kuhns BD, Chahal J, Philippon MJ, Kelly BT, Nho SJ. Hip arthroscopy outcomes with respect to patient acceptable symptomatic state and minimal clinically important difference. Arthroscopy 2016;32:1877-1886.

18. Nasser R, Domb B. Hip arthroscopy for femoroacetabular impingement. EFORT Open Rev 2018;3:121-129.

19. Nwachukwu BU, Chang B, Rotter BZ, Kelly BT, Ranawat AS, Nawabi DH. Minimal clinically important difference and substantial clinical benefit after revision hip arthroscopy. Arthroscopy 2018;34:1862-1868.

20. Almasri M, Simunovic N, Heels-Ansdell D, Ayeni OR, FIRST Investigators. Femoroacetabular impingement surgery leads to early pain relief but minimal functional gains past 6 months: Experience from the FIRST trial. Knee Surg Sports Traumatol Arthrosc 2021;29:1362-1369.

21. Femoroacetabular Impingement Randomized Controlled Trial Investigators, Ayeni OR, Karlsson J, et al. Osteochondroplasty and labral repair for the treatment of young adults with femoroacetabular impingement: A randomized controlled trial. Am J Sports Med 2021;49: 25-34.

22. Nwachukwu BU, Beck EC, Chapman R, Chahla J, Okoroha K, Nho SJ. Preoperative performance of the PROMIS in patients undergoing hip arthroscopic surgery for femoroacetabular impingement syndrome. Orthop J Sports Med 2019;7:2325967119860079.

23. Kollmorgen RC, Hutyra CA, Green C, Lewis B, Olson SA, Mather RC 3rd. Relationship between PROMIS computer adaptive tests and legacy hip measures among patients presenting to a tertiary care hip preservation center. Am J Sports Med 2019;47:876-884.

24. Nwachuwu BU, Rasio J, Beck EC, et al. PROMIS Physical function has a lower effect size and is less responsive than legacy hip specific patient reported outcomes measures following arthroscopic hip surgery. Arthroscopy 2020;36: 2992-2997.

25. Iyer S, Koltsov Jcb, Steinhaus M, et al. A prospective, psychometric validation of national institutes of health patient-reported outcomes measurement information system physical function, pain interference, and upper 
extremity computer adaptive testing in cervical spine patients: Successes and key limitations. Spine (Phila Pa 1976) 2019;44:1539-1549.

26. Tyser AR, Beckmann J, Franklin JD, et al. Evaluation of the PROMIS physical function computer adaptive test in the upper extremity. J Hand Surg Am 2014;39:2047-2051. e2044.

27. Kadri O, Jildeh TR, Meldau JE, et al. How long does it take for patients to complete PROMIS Scores? An assessment of PROMIS CAT questionnaires administered at an ambulatory sports medicine clinic. Orthop J Sports Med 2018;6:2325967118791180.

28. Kwak SK, Kim JH. Statistical data preparation: Management of missing values and outliers. Korean J Anesthesiol 2017;70:407-411.

29. Kortlever JT, Janssen SJ, van Berckel MM, Ring D, Vranceanu AM. What is the most useful questionnaire for measurement of coping strategies in response to nociception? Clin Orthop Relat Res 2015;473:3511-3518.

30. Beckmann JT, Hung M, Bounsanga J, Wylie JD, Granger EK, Tashjian RZ. Psychometric evaluation of the PROMIS Physical Function Computerized Adaptive Test in comparison to the American Shoulder and Elbow Surgeons score and Simple Shoulder Test in patients with rotator cuff disease. J Shoulder Elbow Surg 2015;24: 1961-1967.

31. Morgan JH, Kallen MA, Okike K, Lee OC, Vrahas MS. PROMIS Physical Function Computer Adaptive Test compared with other upper extremity outcome measures in the evaluation of proximal humerus fractures in patients older than 60 years. J Orthop Trauma 2015;29:257-263.

32. Paulino Pereira NR, Janssen SJ, Raskin KA, et al. Most efficient questionnaires to measure quality of life, physical function, and pain in patients with metastatic spine disease: A cross-sectional prospective survey study. Spine J 2017;17:953-961.

33. Segal LS, Plantikow C, Hall R, Wilson K, Shrader MW. Evaluation of patient satisfaction surveys in pediatric orthopaedics. J Pediatr Orthop 2015;35:774-778.
34. Mohebbifar R, Hasanpoor E, Mohseni M, Sokhanvar M, Khosravizadeh O, Mousavi Isfahani H. Outpatient waiting time in health services and teaching hospitals: A case study in Iran. Glob J Health Sci 2013;6:172-180.

35. Levesque J, Bogoch ER, Cooney B, Johnston B, Wright JG. Improving patient satisfaction with time spent in an orthopedic outpatient clinic. Can J Surg 2000;43: 431-436.

36. Patterson BM, Eskildsen SM, Clement RC, et al. Patient satisfaction is associated with time with provider but not clinic wait time among orthopedic patients. Orthopedics 2017;40:43-48.

37. Kittipittayakorn C, Ying KC. Using the integration of discrete event and agent-based simulation to enhance outpatient service quality in an orthopedic department. $J$ Healthe Eng 2016;2016.

38. Domb BG, Battaglia MR, Perets I, et al. Minimum 5-year outcomes of arthroscopic hip labral reconstruction with nested matched-pair benchmarking against a labral repair control group. Am J Sports Med 2019;47:2045-2055.

39. Tevis SE, James TA, Kuerer HM, et al. Patient-reported outcomes for breast cancer. Ann Surg Oncol 2018;25: 2839-2845.

40. Basch E, Barbera L, Kerrigan CL, Velikova G. Implementation of patient-reported outcomes in routine medical care. Am Soc Clin Oncol Educ Book 2018;38: 122-134.

41. Stapleton SJ, Degitz RJ. An innovative data collection method for investigating unresolved pain after ED discharge: A pilot study. J Emerg Nurs 2014;40:598-604.

42. Czaja SJ, Charness N, Fisk AD, et al. Factors predicting the use of technology: Findings from the Center for Research and Education on Aging and Technology Enhancement (CREATE). Psychol Aging 2006;21:333-352.

43. Christopher Leys CL, Klein Oliver, Bernard Philippe, Licata Laurent. Detecting outliers: Do not use standard deviation around the mean, use absolute deviation around the median. J Exp Soc Psychol 2013;49: 764-766. 